\title{
Reduced inertial parameters in system of one degree of freedom obtained by Eksergian's method
}

\author{
Salvador Cardona Foix, Lluïsa Jordi Nebot, and Joan Puig-Ortiz \\ Mechanical Engineering Department, ETSEIB, Universitat Politècnica de Catalunya, Barcelona, 08028, Spain \\ Correspondence to: Lluïsa Jordi Nebot (lluisa.jordi@upc.edu)
}

Received: 23 November 2016 - Revised: 17 February 2017 - Accepted: 9 March 2017 - Published: 30 March 2017

\begin{abstract}
The mechanisms of one degree of freedom can be dynamically analysed by setting out a single differential equation of motion which variable is the generalized coordinate selected as independent. In front of the use of a set of generalized dependent coordinates to describe the system, the method exposed in this work has the advantage of working with a single variable but leads to complex analytical expressions for the coefficients of the differential equation, even in simple mechanisms. The theoretical approach, in this paper, is developed from Eksergian's method and Lagrange's equations. The equation of motion is written by means of a set of parameters - reduced parameters - that characterize the dynamic behaviour of the system. These parameters are function of the independent coordinate chosen and its derivative and can be obtained numerically by direct calculus or by means of a kinetostatic analysis, as is proposed. Two cases of study of the method are presented. The first example shows the study of pedalling a stationary bicycle used in a rehabilitation process. The second one shows the analysis of a single dwell bar mechanism which is driven by an electric motor.
\end{abstract}

\section{Introduction}

Analysis of mechanisms from the kinematic point of view is, in general, not difficult to make except in the singular configurations and their environment. There are a number of programs that facilitate it or directly carry out it in lesser or greater extent: Geogebra (Geogebra, 2016) whose main objective is the dynamic geometry, CAD programs including a kinematic analysis module or a number of specific programs (Kurtenbach et al., 2014). The kinetostatic or inverse dynamic analysis, in which the mechanism has as many actuators as degrees of freedom and all movements are imposed, requires extra effort, both conceptual and operational. This fact is particularly difficult when trying to determine the constraint actions in the kinematic pairs of the mechanism. There are also a number of programs that perform kinetostatic analysis, such as PAM - Program of Analysis of Mechanisms of Mechanical Engineering Department of UPC (Clos and Puig-Ortiz, 2004; Cardona et al., 2006), SAM - Synthesis and Analysis of Mechanisms - by ARTAS Engineering Software (SAM, 2016), WinMecC of Mechanical Engineering Department of Malaga University (WinMecC, 2016) and others. Finally, the direct dynamic analysis, in which the mech- anism has fewer actuators than degrees of freedom, represents a qualitative leap in difficulty of implementation. To the algebraic manipulation of the above analysis the solution of differential equations must be added. There are different applications that carry out direct dynamic analysis. The use of such applications is not always justified because of their complexity and because they are not trivial to use, even for the simulation of a system of one degree of freedom. Their use may be not recommended or even unfeasible in some cases, such as when the study of the mechanism must be performed in real time, as part of the simulation and control of a production process. In these cases, there should be a low-cost model both computational and in implementation.

In the world of robotics it is usual to use reduced inertial parameters to minimize the time of calculation and simulation. Several authors have proposed methods to determine and to reduce the number of parameters to be used in the inverse dynamics of manipulators (Fogarasy and Smith, 1997; Ebrahimi and Haghi, 2013; Díaz-Rodríguez et al., 2010; Ros et al., 2012; Chen and Beale, 2003; Yoshida et al., 1995) as well as to determine the minimum number of required parameters (Gautier and Khalil, 1988). It has also been inves- 
tigated the possibility that the parameters vary over time to facilitate manoeuvrability of manipulators in singular configurations (Parsa et al., 2015). With the aim of describing the inertial behaviour of mechanisms, it seems a good idea to use inertial parameters that vary over time.

The dynamic analysis equations of mechanisms are obtained usually by means of Lagrangian formulation (Fogarasy and Smith, 1997), Newtonian formulation and virtual work principle (Wu et al., 2008).

In this paper, similarly to other works of the authors (Cardona et al., 2009; Jordi et al., 2008), the direct dynamic analysis of a mechanism of one degree of freedom by using its reduced inertial parameters and reduced forces and moments is proposed. Both types of parameters are function of the independent generalized coordinate, taken for kinematic description of the mechanism, and may be obtained, for example, using applications that perform the kinetostatic analysis. With these parameters, the direct dynamic analysis leads to a single second order differential equation, equation of motion, which is easily integrated to obtain the time evolution of the generalized coordinate employed. Each constraint action, force or moment, is given by an algebraic expression that includes the reduced parameters associated with such action and the first two derivatives of the coordinate, obtained independently in the process of integration of the equation of motion. In some of the mentioned works, all the reduced inertial parameters are obtained from the Lagrangian formulation. In this study, these parameters, as well as the reduced forces and moments present in the equation of motion, are introduced from the energy theorem or Eksergian's method (Eksergian, 1930; Doughty, 1988). This approach allows the incorporation, in a simple way conceptually and operationally, of motors and passive resistances described by means of the dissipated energy.

The determination of reduced inertial parameters and reduced forces is performed by using the mentioned programs that allow static, kinematic and kinetostatic analysis of planar mechanisms controlled by actuators, angular or linear.

\section{Dynamics of a mechanism of one degree of freedom}

To obtain the equation of motion of a mechanism of one degree of freedom, the use of energy theorem in differential version or Eksergian's method (Eksergian, 1930) is proposed:

$\dot{E}_{c}=P$

Being $E_{c}$ the kinetic energy of the mechanism and $P$ the sum of the power of the external forces which act on it, and the power of the no-constrain internal forces that make nonull work, as motors or passives resistances, which are not modelled as an explicit function of constrain forces.

For a system of one degree of freedom, kinetic energy $E_{c}$ can be expressed in terms of the independent generalized co- ordinate $q$ and its time derivative, so that:

$E_{c}=\frac{1}{2} m(q) \dot{q}^{2} \rightarrow \dot{E}_{c}=m(q) \ddot{q} \dot{q}+\frac{1}{2} m_{q}(q) \dot{q}^{2} \dot{q}$

Where $m(q)$ is the inertia reduced to the coordinate $q$ and $m_{q}(q)$ its derivative with respect to the coordinate $q$.

The total power $P$ of all the forces acting on the mechanism can be expressed in terms of the reduced force $F(q, \dot{q})$ to the independent coordinate as:

$P=F(q, \dot{q}) \dot{q}$

Thus from Eq. (1), the equation of motion is obtained:

$m(q) \ddot{q}+\frac{1}{2} m_{q}(q) \dot{q}^{2}=F(q, \dot{q})$

For the systems of one degree of freedom and the holonomic ones with more than one degree of freedom described by a set of $n$ independent generalized coordinates, the equations of motion can be obtained by means of the ordinary Lagrange equations:

$\frac{\mathrm{d}}{\mathrm{d} t} \frac{\partial E_{c}}{\partial \dot{q}_{i}}-\frac{\partial E_{c}}{\partial q_{i}}=F_{i}^{*} \quad i=1 \ldots n$

Being $F_{i}^{*}$, the generalized force, associated to the coordinate $q_{i}$, of all forces acting on the mechanism.

By developing Eq. (3), for a system of one degree of freedom, an identical equation to Eq. (2) is obtained. This fact shows that the reduced force and generalized force coincide.

When it is possible to use Eksergian's method, the inclusion of not mechanical phenomena, as motors and passives resistances, in the motion equation is conceptually simple. It can be done by means of the power that these phenomena exchange with the system, and that is described with mechanical state variables. In the Lagrange's formulation, these phenomena must be introduced by means of generalized forces associated to the non-conservative forces.

If in the dynamic study of the mechanism is desired to determine a constraint action, the kinematic condition imposed by the constraint is substitutable, conceptually, by an actuator that ensures it - constraint actuator. Figure 1 shows a four linkage bar mechanism activated by an angular actuator $T_{\text {act }}$ on the crank OP and how to determine the vertical force $F_{e}$ at the $\mathrm{R}$ joint. This joint is replaced by a slider guide which leaves free the vertical movement, which is now constrained with the actuator. Replacing a constraint condition by an actuator, although conceptually, means that the initial mechanism of one degree of freedom must be studied as a new holonomic mechanism of two degrees of freedom with two independent coordinates $q_{1}$ and $q_{2}$. The first coordinate $q_{1}$ associated with the actual movement of the mechanism and the second $q_{2}$ with the movement prevented by the constraint. Thus, the motion equation describing temporal evolution of $q_{1}$ can be determined by Eq. (2) obtained with Eksergian's 


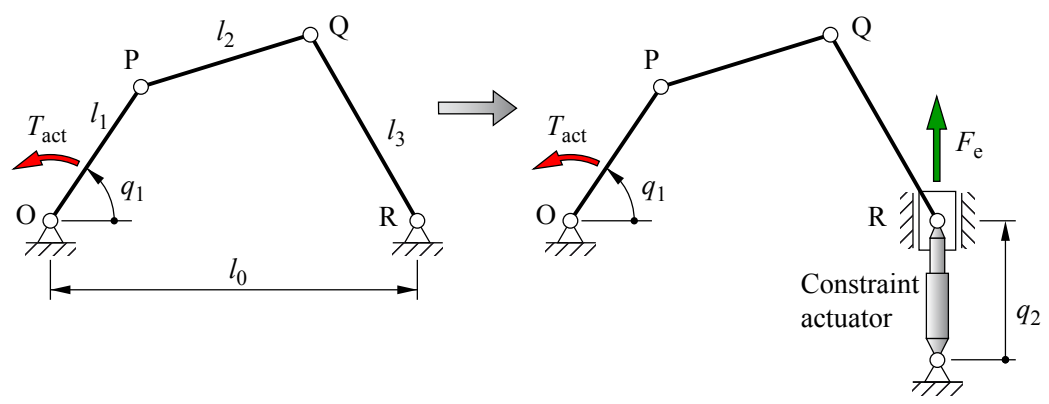

Figure 1. Original mechanism and with a constraint condition replaced by a constraint actuator.

method. The constraint force or moment can be determined with the obtained expressions from Lagrange's method.

For the new system of two degrees of freedom, the kinetic energy is (Jordi et al., 2008; Eksergian, 1930; Cardona and Clos, 2000):

$$
\begin{aligned}
E_{c} & =\frac{1}{2} m_{1}\left(q_{1}, q_{2}\right) \dot{q}_{1}^{2}+\frac{1}{2} m_{2}\left(q_{1}, q_{2}\right) \dot{q}_{2}^{2} \\
& +m_{12}\left(q_{1}, q_{2}\right) \dot{q}_{1} \dot{q}_{2}
\end{aligned}
$$

Applying Lagrange equations to this mechanism, and particularizing for the movement imposed by the constraint actuator $\ddot{q}_{2}=0$ and $\dot{q}_{2}=0$ the algebraic equation including the generalized force $F_{2}^{*}$ is obtained. It contains, besides the forces of known formulation, the constraint force or moment desired. In summary, for the example of the original mechanism of Fig. 1 the equation of motion obtained with Eq. (2) is:

$m_{1}\left(q_{1}\right) \ddot{q}_{1}+\frac{1}{2} m_{1 q_{1}}\left(q_{1}\right) \dot{q}_{1}^{2}=T_{\mathrm{act}}$

In general, $T_{\text {act }}$ is the reduced force associated to the coordinate $q_{1}$ of all the forces different from the constraint forces.

The constraint force obtained with Eq. (3) is:

$m_{12}\left(q_{1}\right) \ddot{q}_{1}+\left(m_{12 q_{1}}\left(q_{1}\right)-\frac{1}{2} m_{1 q_{2}}\left(q_{1}\right)\right) \dot{q}_{1}^{2}=F_{2}^{*}$

Being $m_{1 q_{1}}=\frac{\partial m_{1}}{\partial q_{1}} ; m_{12 q_{1}}=\frac{\partial m_{12}}{\partial q_{1}} ; m_{1 q_{2}}=\frac{\partial m_{1}}{\partial q_{2}}$ and $F_{2}^{*}=F_{e}$.

The coefficients of the Eqs. (5) and (6), which are reduced inertial parameters, can be determined from the calculation of the kinetic energy or, as it will be discussed below, using kinetostatic simulation programs as PAM, SAM or WinMecC.

The kinetostatic analysis allows to determine $T_{\text {act }}$ given $q(t)$. Taking the system of one degree of freedom of Fig. 1 as an example, to determine the reduced parameters of the equation of motion the following kinetostatic analyses are performed:

i. $\ddot{q}_{1}=0$ and $\dot{q}_{1} \neq 0$; so $m_{1 q_{1}}\left(q_{1}\right)=2 T_{\text {act }} / \dot{q}_{1}^{2}$ is obtained.

ii. $\ddot{q}_{1} \neq 0$ and $\dot{q}_{1}=0$;

so $m_{1}\left(q_{1}\right)=\left(T_{\text {act }}-\frac{1}{2} m_{1 q_{1}}\left(q_{1}\right) \dot{q}_{1}^{2}\right) / \ddot{q}_{1}$ is obtained.
The parameters of the Eq. (6) are obtained by the kinetostatic analyses of the same system, so that:

$$
\begin{aligned}
& \text { i. } \ddot{q}_{1}=0 \text { and } \dot{q}_{1} \neq 0 \text {, } \\
& m_{e 1_{q}}\left(q_{1}\right)=\left(m_{12 q_{1}}\left(q_{1}\right)-\frac{1}{2} m_{1 q_{2}}\left(q_{1}\right)\right)=F_{2}^{*} / \dot{q}_{1}^{2} .
\end{aligned}
$$

ii. $\ddot{q}_{1} \neq 0$ and $\dot{q}_{1}=0, m_{e 1}\left(q_{1}\right)=m_{12}\left(q_{1}\right)$

$$
=\left(F_{2}^{*}-\left(m_{12 q_{1}}\left(q_{1}\right)-\frac{1}{2} m_{1 q_{2}}\left(q_{1}\right)\right) \dot{q}_{1}^{2}\right) / \ddot{q}_{1} .
$$

With programs as PAM, the first analysis is easily implemented using an actuator with a polynomial movement law and imposing the above condition with constant velocity $\left(\ddot{q}_{1}=0\right.$ and $\left.\dot{q}_{1} \neq 0\right)$. This analysis is performed for a set of uniformly distributed instants of time and thus sweep the entire range of values of interest of $q_{1}$. So, values of $m_{1 q_{1}}$ and $m_{e 1_{q}}$ are obtained for values of $q_{1}$ equispaced.

The necessary conditions of the second analysis $\left(\ddot{q}_{1} \neq 0\right.$ and $\dot{q}_{1}=0$ ) are impossible to perform with an actuator with a polynomial movement law. So, if you want to get the values of $m_{1}$ and $m_{e 1}$ for the same values of $q_{1}$ of the first analysis, a strategy should be used. This may be the use of an actuator that controls $q_{1}$ according to a temporal function with two parts: one polynomial and another one harmonic, so that:

$q_{1}(t)=\left(c_{1}+c_{2} t\right)+\left(c_{3} \cos \left(c_{4} t+c_{5}\right)\right)$

where $c_{1} \ldots c_{5}$ are constants which are chosen so that the conditions $\ddot{q}_{1} \neq 0$ and $\dot{q}_{1}=0$ occur at points of interest. In short, a continuous function for the actuator is defined and properly sampled provides the required conditions in the desired $q_{1}$ configurations.

SAM has a utility that facilitates the realization of the two analyses evaluated at the same instants of time. You can perform a kinetostatic analysis by loading a file in which the temporary values, the value of the coordinate $q_{1}$ and its first two derivatives are specified. So, for doing the two analyses two input files have to be created. These files must contain, for the range of values of interest of $q_{1}$, the values of this variable equally spaced at time regular intervals. In the first file, these values are associated to a speed $\dot{q}_{1}=1$ and acceleration $\ddot{q}_{1}=0$, and in the second file these values are associated to a speed $\dot{q}_{1}=0$ and acceleration $\ddot{q}_{1}=1$. The values of speed and acceleration have the default units used in the analysis. 


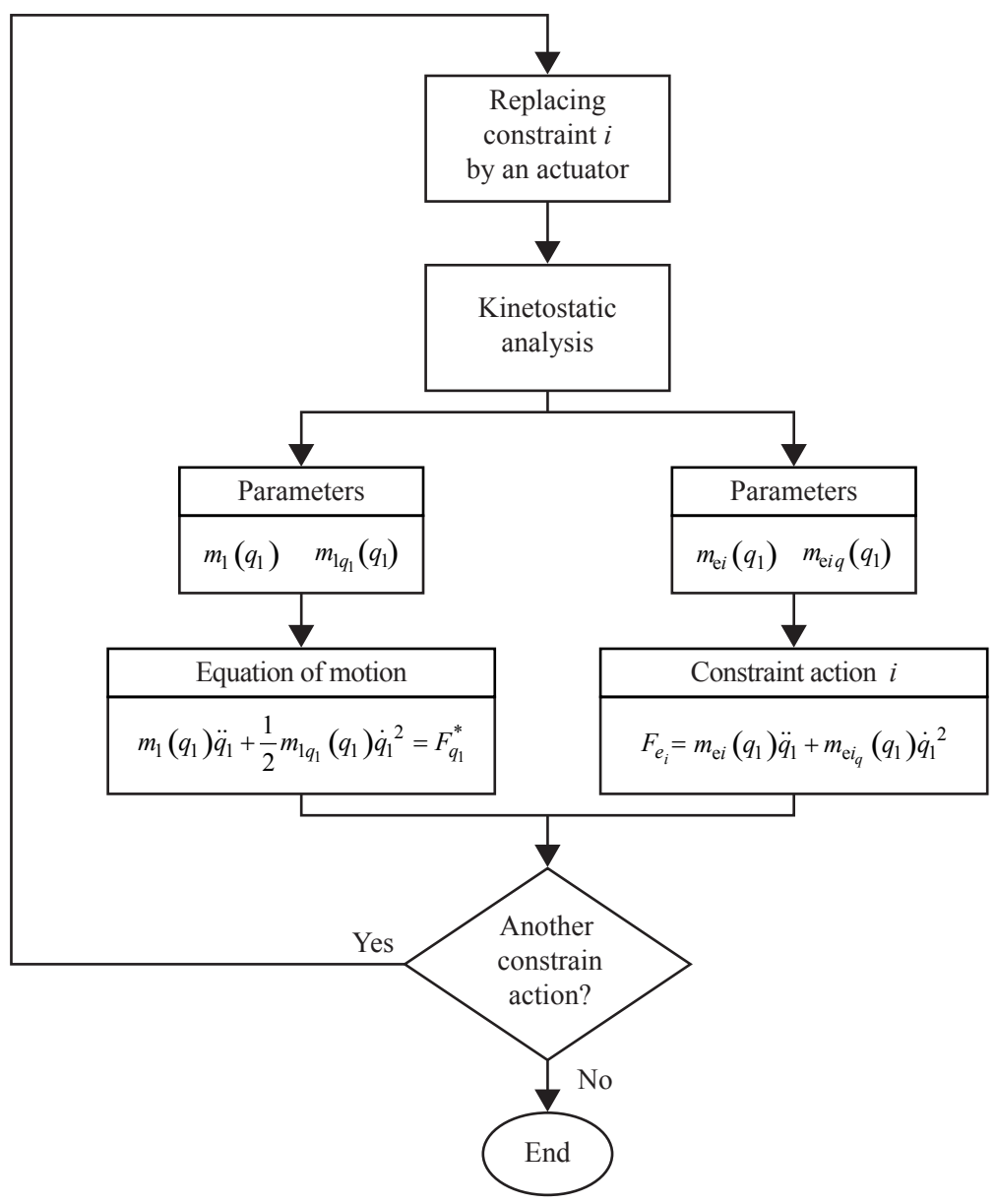

Figure 2. Proposed process for dynamic analysis of a system of one degree of freedom.

The process presented for the determination of the constraint action is extensible to find simultaneously as many actions as desired. In this case, as many constraint actuators as constraint actions to determine must be defined to obtain the corresponding equations. In order not to increase the complexity of the system, as many independent systems of two degrees of freedom as constraint actions to determine must be defined. Each system provides an equation for the constraint action and an equation of motion, that is obviously the same for all systems. Thus, two reduced parameters for the equation of motion and two parameters for each constraint action to determine are obtained. Figure 2 shows a schematic of the process to be followed for determining the constraint actions desired.

\section{Cases of study}

The procedure described in the previous section is used to study two cases: the pedalling a stationary bicycle used in a rehabilitation process and the analysis of a single dwell bar mechanism which is driven by an electric motor.

\subsection{Pedalling a stationary bicycle used in a rehabilitation process of knee damage}

The dynamics of the pedalling in a stationary bicycle in a rehabilitation process (Cardona et al., 2009; Jordi et al., 2008; Curià, 2010) is studied. The mechanism of Fig. 3 represents the model for this study. It is assumed that the ankle is fixed to the pedal, so the model has only one degree of freedom. The geometric characteristics and inertial parameters, obtained experimentally by Curià (2010), are shown in Fig. 3a. The translation kinetic energy of the bicycle and the cyclist plus the rotation kinetic energy of the wheels of a conventional bicycle are substituted by the kinetic energy of a flywheel fixed to the pinion. Some aspects as the action of several muscles, that can be modelled as simultaneous actuators, are not taken into account.

The most reasonable kinetostatic analysis to determine reduced parameters is the one that uses an actuator controlling the rotation angle of the pedals $\varphi_{\mathrm{p}}$ because this coordinate does not have dead-points (Fig. 3b). In this analysis, the variations of potential energy associated to the thigh and the leg are not taken into account. 

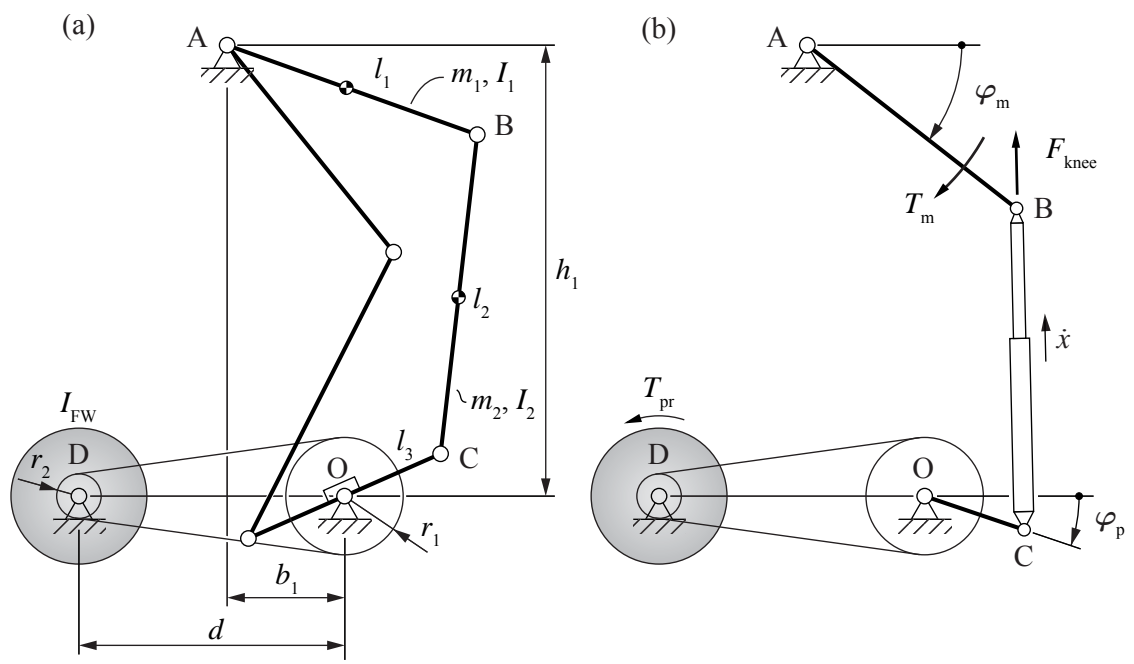

$\begin{array}{ll}h_{1}=730 \mathrm{~mm} & m_{1}=6.5 \mathrm{~kg} \\ b_{1}=190 \mathrm{~mm} & I_{1}=0.0871 \mathrm{~kg} \mathrm{~m}^{2} \\ d=430 \mathrm{~mm} & m_{2}=4.2 \mathrm{~kg} \\ l_{1}=430 \mathrm{~mm} & I_{2}=0.0905 \mathrm{~kg} \mathrm{~m}^{2} \\ l_{2}=520 \mathrm{~mm} & I_{\mathrm{FW}}=10 \mathrm{~kg} \mathrm{~m}^{2} \\ l_{3}=170 \mathrm{~mm} & \\ r_{1}=95 \mathrm{~mm} \text { (47 teeth) } \\ r_{2}=36 \mathrm{~mm} \text { (18 teeth) }\end{array}$

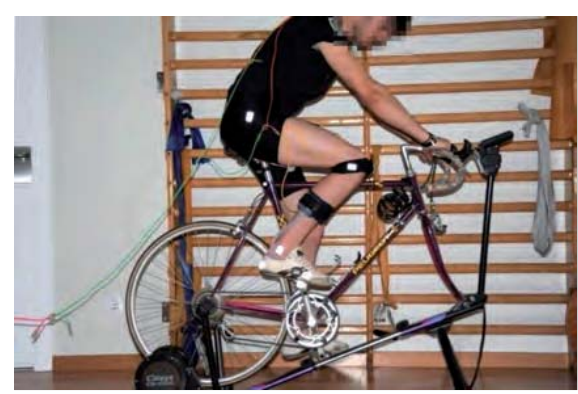

Figure 3. Model for the stationary bicycle and the inferior extremities of the cyclist.

The motion equation of the system of Fig. 3a can be obtained by means of Eksergian's method. The kinetic energy of the system is:

$$
\begin{aligned}
E_{c} & =\frac{1}{2}\left(I_{1}+m_{1}\left(\frac{l_{1}}{2}\right)^{2}\right)\left(\dot{\varphi}_{\mathrm{m} 1}^{2}+\dot{\varphi}_{\mathrm{m} 2}^{2}\right)+\frac{1}{2} I_{\mathrm{FW}}\left(\frac{r_{1}}{r_{2}}\right)^{2} \dot{\varphi}_{\mathrm{p}}^{2} \\
& +\frac{1}{2} m_{2}\left(v_{\mathrm{G} 21}^{2}+v_{\mathrm{G} 22}^{2}\right)+\frac{1}{2} I_{2}\left(\omega_{21}^{2}+\omega_{22}^{2}\right)
\end{aligned}
$$

Where $\dot{\varphi}_{\mathrm{m} 1}$ and $\dot{\varphi}_{\mathrm{m} 2}$ are the angular velocities of the two thighs, $\dot{\varphi}_{\mathrm{p}}$ is the angular velocity of the pedals, $v_{\mathrm{G} 21}$ and $v_{\mathrm{G} 22}$ are the velocities of the centre of mass of the legs and $\omega_{21}$ and $\omega_{22}$ are the angular velocities of the legs. Obviously, all these kinematics variables are related by means of the kinematic constrain equations. With this approach, the motion equation can be obtained. If an additional objective of the analysis is to determine some constrain action it is necessary to use Eq. (6) that has been demonstrated with Lagrange's method. It is interesting to remark that the proposed method consists of replacing a constrain by an actuator instead of using Lagrange multipliers method.

The objectives of this analysis are to determine the motion equation and the force in the knee in the leg direction $F_{\text {knee }}$ when a torque of passive resistances $T_{\mathrm{pr}}$ is acting on the flywheel. So, it is necessary to obtain the generalized forces $F_{\varphi_{\mathrm{p}}}^{*}$ and $F_{x}^{*}$ associated to the rotation of the pedal $\varphi_{\mathrm{p}}$ and to the extension $x$ of the constraint actuator.

The expressions of generalized forces are obtained, for example, by means of the following virtual movements:

i. $\dot{\varphi}_{\mathrm{p}}^{*} \neq 0$ and $\dot{x}^{*}=0$. This virtual movement is compatible with the constraints of the original system. The relationship between velocities is obtained from the kinematic analysis of the initial mechanism, that is the same as the mechanism with the constrain actuator with $\dot{x}=0$. The generalized force is:

$$
F_{\varphi_{\mathrm{p}}}^{*}=\left.T_{\mathrm{m}} \frac{\dot{\varphi}_{\mathrm{m}}}{\dot{\varphi}_{\mathrm{p}}}\right|_{\dot{x}=0}+\left.T_{\mathrm{pr}} \frac{\dot{\varphi}_{\text {flywheel }}}{\dot{\varphi}_{\mathrm{p}}}\right|_{\dot{x}=0} .
$$

ii. $\dot{\varphi}_{\mathrm{p}}^{*}=0$ and $\dot{x}^{*} \neq 0$. The generalized force is:

$$
F_{x}^{*}=F_{\mathrm{knee}}+\left.T_{\mathrm{m}} \frac{\dot{\varphi}_{\mathrm{m}}}{\dot{x}}\right|_{\dot{\varphi}_{\mathrm{p}}=0} .
$$

In this movement, the mechanism has two degrees of freedom because the restriction $x=l_{2}$ is not considered. The relationship between velocities is obtained for a movement of the mechanism with the constrain actuator with $\dot{\varphi}_{\mathrm{p}}=0$. 

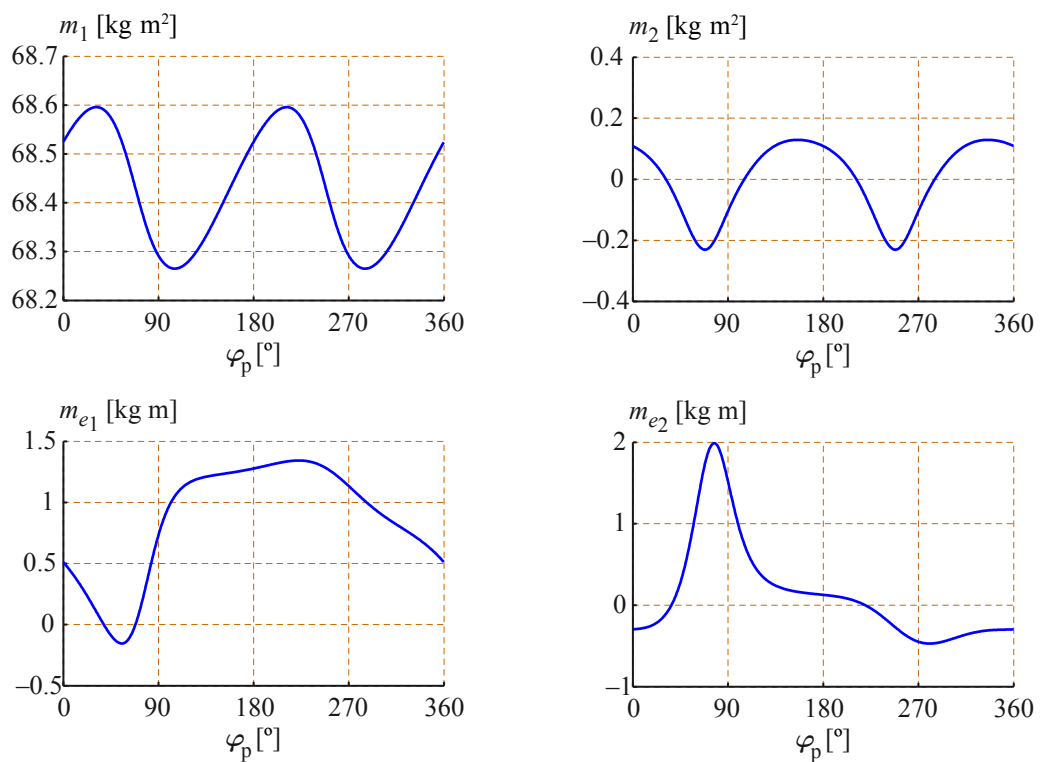

Figure 4. Inertial reduced parameters for pedalling a stationary bicycle.
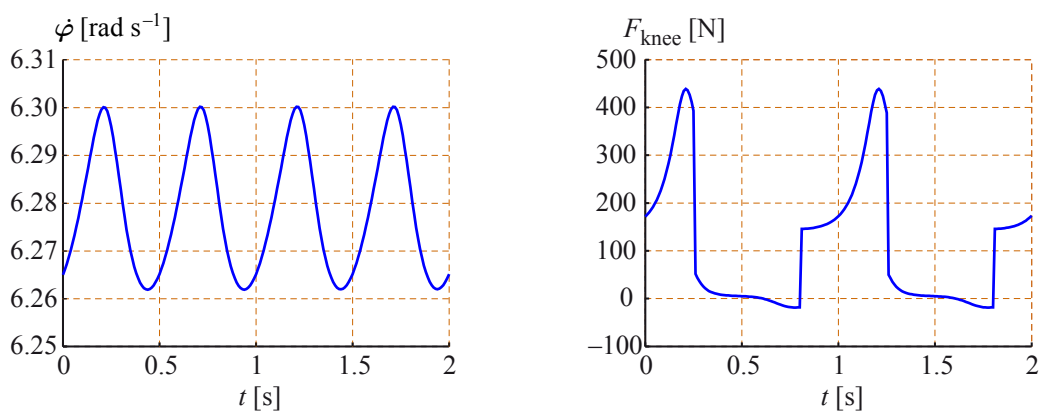

Figure 5. Angular velocity of the pedal and force in the knee in leg direction.

The terms $\frac{\dot{\varphi}_{\mathrm{m}}}{\dot{\varphi}_{\mathrm{p}}}, \frac{\dot{\varphi}_{\text {flywheel }}}{\dot{\varphi}_{\mathrm{p}}}$ and $\frac{\dot{\varphi}_{\mathrm{m}}}{\dot{x}}$ are obtained directly from the kinematic analysis of the mechanism with the corresponding conditions: only one generalized velocity not null.

In order to finish the analysis, the equations have to be solved; so it is necessary to obtain the values of $T_{\mathrm{m}}$ and $T_{\mathrm{pr}}$. The torque $T_{\mathrm{pr}}$, introduced by the dynamic brake of the stationary bicycle, is assumed as constant and its value has been calculated in order that lost power will be $125 \mathrm{~W}$, when the pedalling rhythm is $1 \mathrm{~Hz}$. The torque $T_{\mathrm{m}}$, applied to each thigh, is assumed as constant and not null only in the descent phase of the movement of the thigh. Its value has been determined using the described procedure in order to achieve a pedalling stationary regime with the torque $T_{\mathrm{pr}}$ previously obtained. The value obtained is $T_{\mathrm{m}}=69.7 \mathrm{~N} \mathrm{~m}$. The equations to solve are:

$\left\{\begin{array}{l}m_{1}\left(\varphi_{\mathrm{p}}\right) \ddot{\varphi}_{\mathrm{p}}+m_{2}\left(\varphi_{\mathrm{p}}\right) \dot{\varphi}_{\mathrm{p}}^{2}=F_{\varphi_{\mathrm{p}}}^{*} \\ m_{e 1}\left(\varphi_{\mathrm{p}}\right) \ddot{\varphi}_{\mathrm{p}}+m_{e 2}\left(\varphi_{\mathrm{p}}\right) \dot{\varphi}_{\mathrm{p}}^{2}=F_{x}^{*}\end{array}\right.$
Figure 4 shows the inertial reduced parameters for one revolution of the pedal obtained by means of the exposed procedure of Sect. 2. Calculations have been made with Scilab and simulations with PAM and WinMecC. The results from the simulation have been obtained with intervals of $10^{\circ}$ which are sufficient due to the form of the functions. For their use in Scilab, polynomial functions defined with splines of third order have been used.

Figure 5 shows the rotation velocity of the pedal obtained by means of the integration of the equation of movement. Its mean value remains nearly constant because $m_{1}\left(\varphi_{\mathrm{p}}\right)$ has higher values in front of $m_{2}\left(\varphi_{\mathrm{p}}\right)$. The figure also shows the force in the knee in the leg direction. The low values of the force correspond when the leg is driven, the high values correspond when the leg is the driving one and the higher value corresponds to the dead-point $\varphi_{m}$. 


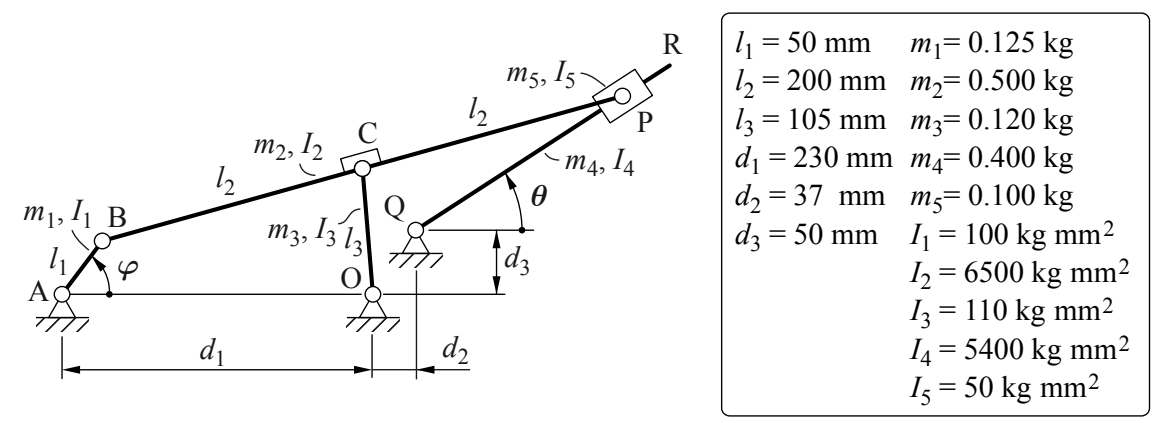

Figure 6. Sketch of the single dwell bar mechanism used in the case of study.

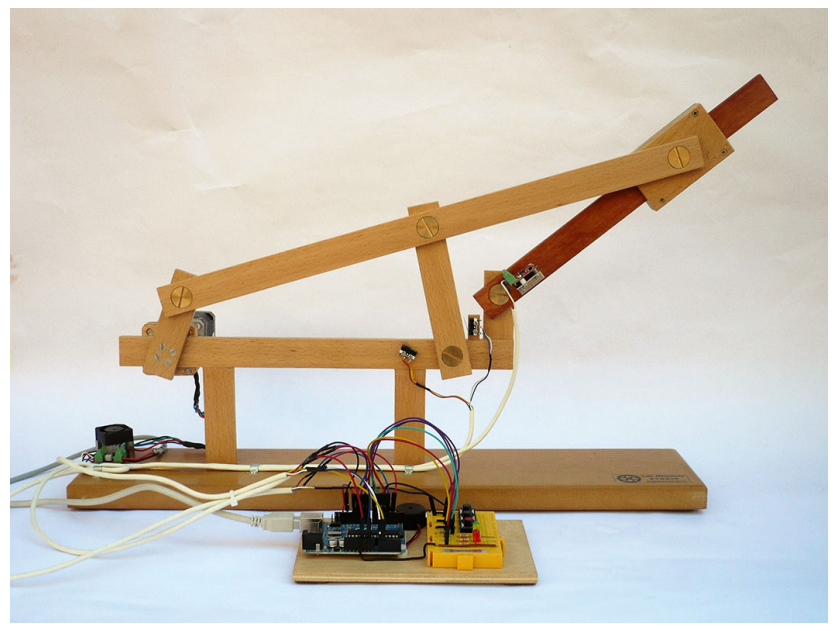

Figure 7. Prototype of the single dwell bar mechanism.

\subsection{Single dwell bar mechanism}

The procedure described in the Sect. 2 is used to study the dynamics of a single dwell bar mechanism. The mechanism and its characteristics, both geometric and inertial, are shown in Fig. 6 which represents the model for this study. The prototype shown in Fig. 7 is designed, among other functions, to check the temporal movement law of the crank $\mathrm{AB}, \varphi(t)$, in order to achieve the prescribed movement law of the rocker QR, $\theta(t)$. A dual axis gyroscope IOG 500 is attached to the rocker to check the accuracy of the result. Even though, in this work, the exposed method in Sect. 2 is used to determine the reduced parameters of this mechanism.

The orientation of the rocker QR is maintained substantially constant for a certain interval of movement of the crank $\mathrm{AB}$. The crank $\mathrm{AB}$ is considered balanced so that its centre of inertia coincides with the fixed joint $\mathrm{A}$. The centre of inertia of the connecting-rod BP is at $\mathrm{C}$ and the centres of inertia of the rockers $\mathrm{OC}$ and $\mathrm{QR}$ are at their midpoint. The centre of inertia of the slider coincides with the articulation P.

The motion equation of the system of Fig. 6 can be obtained by means of Eksergian's method. The kinetic energy of the system is the sum of the kinetic energies of the crank

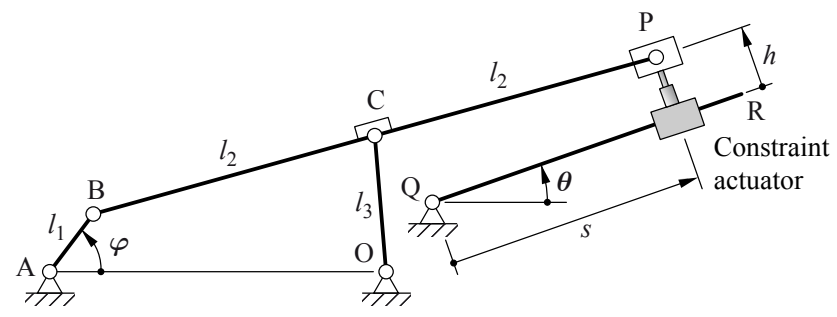

Figure 8. Prototype of the single dwell bar mechanism.

$\mathrm{AB}$, the connecting-rod $\mathrm{BP}$, the two rockers $\mathrm{OC}$ and $\mathrm{QR}$ and the slider. As in the previous example, if some constrain action must be determined it is necessary to use the same procedure of the previous example.

Also in this case, first of all, reduced parameters are determined by kinetostatic simulation programs mentioned and, for a set of time instants, the kinematic and dynamic variables for calculating inertial parameters and reduced forces are obtained. The actuators used in these analyses are an angular actuator that controls the angle $\varphi$ rotated by the crank $\mathrm{AB}$ and a constraint actuator in the prismatic pair as shown in Fig. 8.

From these analyses, the inertial behaviour of the mechanism is obtained. This must be linked with the external forces acting on it, that is the torque of the motor applied in the crank AB $T_{\text {motor }}$ and of the passive resistances $T_{\mathrm{pr}}$. In order to take into account these external forces, the generalized forces associated to the coordinates corresponding to the crank angle and the displacement of the constraint actuator must be calculated. The expressions of generalized forces $F_{i}^{*}$ are obtained by means of the following virtual movements:

i. $\dot{\varphi}^{*} \neq 0$ and $\dot{h}^{*}=0$. This virtual movement is compatible with the constraints of the original system. For this virtual movement the generalized force $F_{\varphi}^{*}$ is obtained:

$F_{\varphi}^{*}=T_{\text {motor }}+T_{\mathrm{pr}} \frac{\partial \dot{\theta}}{\partial \dot{\varphi}}$ 

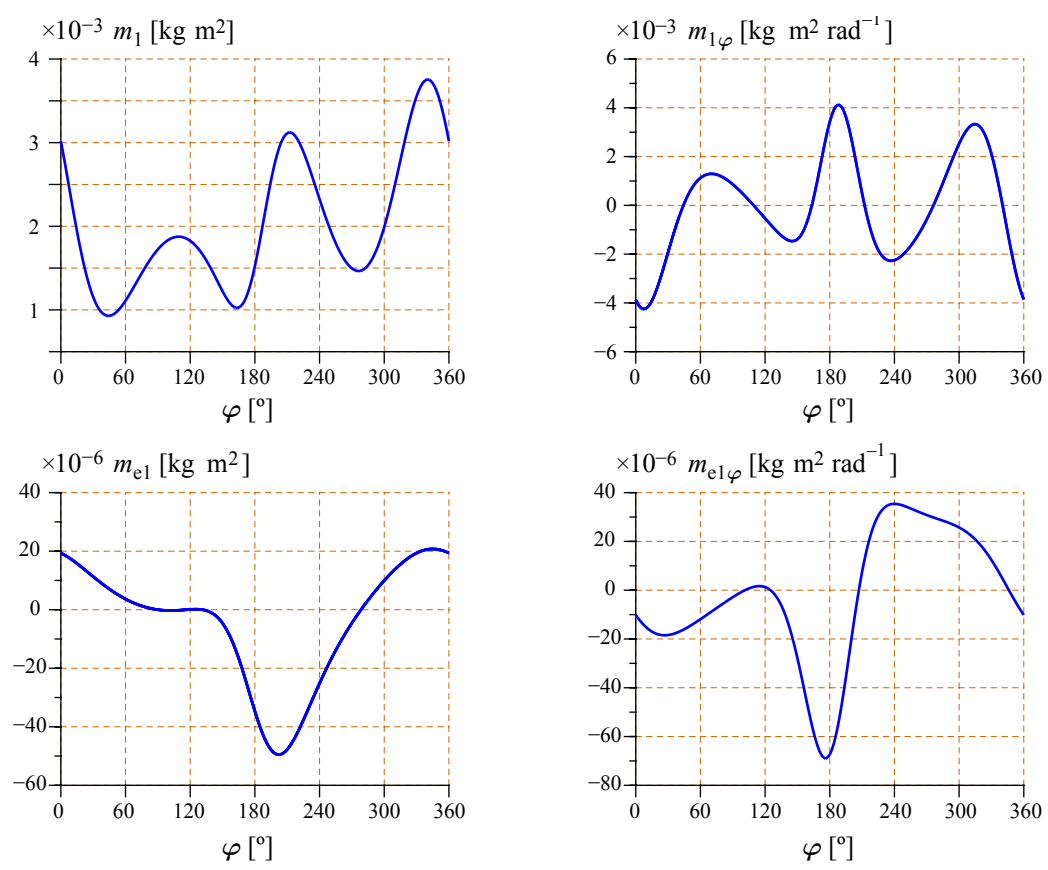

Figure 9. Reduced parameters of the single dwell bar mechanism.
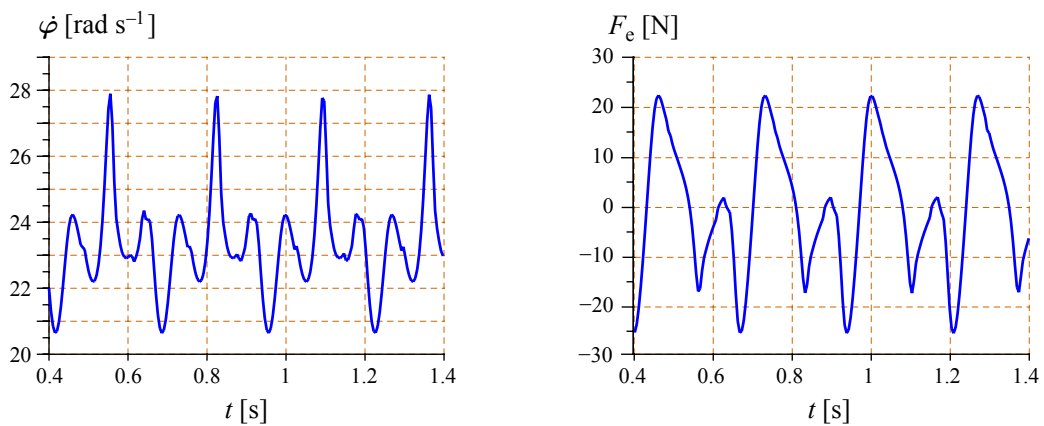

Figure 10. Angular velocity of the crank $\mathrm{AB}$ and constraint force in the prismatic pair.

ii. $\dot{\varphi}^{*}=0$ and $\dot{h}^{*} \neq 0$. For this virtual movement the generalized force $F_{h}^{*}$ is obtained:

$$
F_{h}^{*}=T_{\mathrm{pr}} \frac{\partial \dot{\theta}}{\partial \dot{h}}+F_{e}
$$

The terms $\frac{\partial \dot{\theta}}{\partial \dot{\varphi}}$ and $\frac{\partial \dot{\theta}}{\partial \dot{h}}$ are obtained directly from the kinematic analysis of the mechanism.

The differential equation of movement for the coordinate $\varphi$ that must be integrated is:

$m_{1}(\varphi) \ddot{\varphi}+m_{1 \varphi}(\varphi) \dot{\varphi}^{2}=F_{\varphi}^{*}$

The equation for finding the constraint force in the prismatic pair, from the values of $\varphi$ obtained by means of the integration of Eq. (8) and its derivatives, is:

$m_{e 1}(\varphi) \ddot{\varphi}+m_{e 1 \varphi}(\varphi) \dot{\varphi}^{2}=F_{h}^{*}$
The motor used to determine the reduced parameters is a DC motor with permanent magnets. The operation of this type of motors can be described by the following equations:

$U=R i+L i+K \dot{\varphi}_{\text {motor }}$

$T_{\text {motor }}=K i$

where $U$ is the tension of the armature, $i$ is the intensity that flows through it, $R$ and $L$ are the resistance and inductance in terminals of the armature, $K$ is the constant of the torque, $T_{\text {motor }}$ is the generated torque and $\dot{\varphi}_{\text {motor }}$ is the angular velocity of the motor.

The motor chosen in this application is a model Dunkermotoren GR $63 \times 55$ powered $12 \mathrm{~V}$ with the following characteristics: $R=0.6 \Omega, L=1.5 \mathrm{mH}, K=64 \mathrm{mN} \mathrm{m} \mathrm{A}^{-1}$, $I_{\mathrm{mot}}=75 \mathrm{~kg} \mathrm{~mm}^{2}$. In order to adjust the velocity of rotation of the motor to that necessary in the entrance shaft of the mechanism, the use of a gear reducer is proposed. The cho- 
sen one is PLG 52 of the same brand with a transmission ratio $\tau_{\text {red }}=0.125$ and an inertia momentum reduced to the entrance shaft $I_{\text {red }}=20 \mathrm{~kg} \mathrm{~mm}^{2}$.

The passive resistances have been considered concentrated in bar QR and are modelled as:

$T_{\mathrm{rp}}=-T_{0} \operatorname{sign}(\theta)-c \dot{\theta}$

where $T_{0}=0.1 \mathrm{~N} \mathrm{~m}$ and $c=0.03 \mathrm{~N} \mathrm{~m}\left(\mathrm{rad} \mathrm{s}^{-1}\right)^{-1}$.

Figure 9 shows the inertial reduced parameters for one revolution of the crank $\mathrm{AB}$ obtained by means of the exposed procedure of Sect. 2. Calculations have been made with Scilab and simulations with PAM and SAM. All the results agree among them.

Figure 10 shows the rotation velocity of the crank obtained by means of the integration of Eq. (8) and the constraint force in the prismatic pair obtained with Eq. (9). Rotation velocity and force are periodic. Although the value of motor torque is almost constant the considerable variation of the rotation velocity is consequence of the great variation of the inertial parameters.

\section{Conclusions}

The proposed approach for doing the dynamic analysis of one degree of freedom mechanisms (not necessary planar) based on the use of reduced parameters leads to a simple and efficient procedure for their study.

This approach enlarges the possibilities and utilities of easy use programs that cannot do direct dynamic analysis. In particular, the inertial reduced parameters have been obtained with programs PAM, SAM and WinMecC and the reduced parameters for calculating the generalized forces with PAM.

This approach is useful when the study of the mechanism must be performed in real time, as part of the simulation and control of a production process. A comparison between the time used to integrate two cycles of stationary regime of the example 1 with a commercial software and with the proposed method has been performed. The computation time on a PC with Windows7 operating system and 64-bit processor Intel Core i7 have been $658 \mathrm{~ms}$ with commercial software and $68 \mathrm{~ms}$ with the proposed method. The results support the statement that this method can be used in real-time simulations more efficiently.

The inertial reduced parameters are obtained as a table of values. So, the integration algorithm for the equation of movement must access to them by means of an interpolation function.

The use of Eksergian's method allows the incorporation, in a simple way conceptually and operationally, of motors and passive resistances described by means of the exchanged energy, instead of doing it by means of non-conservative forces.

The exposed procedure is easily extensible to conservative forces, that only depend on position. The generalized forces associated to those can be obtained by kinetostatic analysis. Forces that depend on velocity must be dealt directly in the equation of movement. This type of forces can also be reduced to the coordinate, but the associated generalized force is, then, function of position and velocity and its determination by means of a kinetostatic analysis is not operative.

Data availability. All the data used in this manuscript can be obtained by requesting from the corresponding author.

Competing interests. The authors declare that they have no conflict of interest.

Edited by: A. Müller

Reviewed by: two anonymous referees

\section{References}

Cardona, S. and Clos, D.: Teoria de Màquines, UPC, Barcelona, 2000.

Cardona, S., Clos, D., Jordi, L., and Puig-Ortiz, J.: Curs d'autoaprenentatge de simulació de mecanismes, Universitat Politècnica de Catalunya, Barcelona, 2006.

Cardona, S., Jordi, L., and Puig-Ortiz, J.: Utilización de fuerzas y parámetros reducidos para el estudio dinámico de mecanismos de un grado de libertad, Congreso Iberoamericano de Ingeniería Mecánica, 17-20 November 2009, Las Palmas de Gran Canaria, Spain, 1503-1510, 2009.

Chen, K. and Beale, D. G.: A linear approach for experimental dynamic parameter estimation of planar mechanisms, Multibody Syst. Dyn., 9, 165-184, doi:10.1023/A:1022552113353, 2003.

Clos, D. and Puig-Ortiz, J.: PAM, un programa de análisis de mecanismos planos de $\mathrm{n}$ grados de libertad enfocado a la docencia universitaria, An. Ing. Mec., 15, 757-765, 2004.

Curià, E.: Una modificació de la pedalada. Estudi mecànic i biomecànic. Repercussió en la limitació de la flexió del genoll, PhD Thesis, Universitat de Lleida, Spain, 2010.

Díaz-Rodríguez, M., Mata, V., Valera, A., and Page, A.: A methodology for dynamic parameters identification of 3-DOF parallel robots in terms of relevant parameters, Mech. Mach. Theory, 45, 1337-1356, doi:10.1016/j.mechmachtheory.2010.04.007, 2010.

Doughty, S.: Mechanics of Machines, John Wiley \& Sons, USA, 1988.

Ebrahimi, S. and Haghi, A.: Characterization of the contribution of inertial parameters to the dynamics of multibody systems, Multibody Syst. Dyn., 30, 449-460, doi:10.1007/s11044-013-9355-x, 2013.

Eksergian, R.: Dynamical Analysis of Machines, J. Franklin I., 209, 21-36, doi:10.1016/S0016-0032(30)90992-1, 1930.

Fogarasy, A. A. and Smith, M. R.: A unified tensor approach to the analysis of mechanical systems, P. I. Mech. Eng. C-J. Mec., 211, 313-322, doi:10.1243/0954406971522079, 1997.

Gautier, M. and Khalil, W.: A direct determination of minimum inertial parameters of robots, 27th IEEE Conference on Decision and Control, 7-9 December 1988, Austin, Texas, 1682-1687, doi:10.1109/CDC.1988.194738, 1988. 
Geogebra: available at: http://www.geogebra.org, last access: 25 October 2016.

Jordi, L., Zayas, E. E., and Cardona, S.: Reduced parameters for the dynamic study of a system of one degree of freedom, 12th International Research/Expert Conference "Trends in the Development of Machinery and Associated Technology", 26-30 August 2008, Istanbul, Turkey, 1049-1052, 2008.

Kurtenbach, S., Prause, I., Weigel, C., and Corves, B.: Comparison of Geometry Software for the Analysis in Mechanism Theory, in: New Trends in Educational Activity in the Field of Mechanism and Machine Theory, Springer International Publishing, Switzerland, 193-201, 2014.

Parsa, S. S., Boudreau, R., and Carretero, J. A.: Reconfigurable mass parameters to cross direct kinematic singularities in parallel manipulators, Mech. Mach. Theory, 85, 53-63, doi:10.1016/j.mechmachtheory.2014.10.008, 2015.

Ros, J., Iriarte, X., and Mata, V.: 3D inertia transfer concept and symbolic determination of the base inertial parameters, Mech. Mach. Theory, 49, 284-297, doi:10.1016/j.mechmachtheory.2011.09.006, 2012.
SAM (Mechanism Design Software, Kinematics, Simulation, Optimization): available at: http://www.artas.nl, last access: 25 October 2016.

WinMecC (Programa de análisis cinemático y dinámico de mecanismos planos): available at: http://winmecc.uma.es, last access: 25 October 2016.

Wu, J., Wang, J., Wang, L., and Shao, H.: Dimensional synthesis and dynamic manipulability of a planar two-degree-of-freedom parallel manipulator, P. I. Mech. Eng. C-J Mec., 222, 1061-1069, doi:10.1243/09544062JMES830, 2008.

Yoshida, K., Mayeda, H., and Ono, T.: Base parameters for manipulators with a planar parallelogram link mechanism, Adv. Robotics, 10, 105-137, doi:10.1163/156855396X00147, 1995. 